\title{
Pollinator-Driven Speciation in Sexually Deceptive Orchids
}

\author{
Shuqing Xu, ${ }^{1,2}$ Philipp M. Schlüter, ${ }^{1}$ and Florian P. Schiestl ${ }^{1}$ \\ ${ }^{1}$ Institute of Systematic Botany, University of Zürich, Zollikerstrasse 107, 8008 Zürich, Switzerland \\ ${ }^{2}$ Department of Molecular Ecology, Max Planck Institute for Chemical Ecology, 07745 Jena, Germany \\ Correspondence should be addressed to Shuqing Xu, sxu@ice.mpg.de
}

Received 23 July 2011; Accepted 8 November 2011

Academic Editor: Rui Faria

Copyright () 2012 Shuqing Xu et al. This is an open access article distributed under the Creative Commons Attribution License, which permits unrestricted use, distribution, and reproduction in any medium, provided the original work is properly cited.

\begin{abstract}
Pollinator-mediated selection has been suggested to play a major role for the origin and maintenance of the species diversity in orchids. Sexually deceptive orchids are one of the prime examples for rapid, pollinator-mediated plant radiations, with many species showing little genetic differentiation, lack of postzygotic barriers, but strong prezygotic reproductive isolation. These orchids mimic mating signals of female insects and employ male insects as pollinators. This kind of sexual mimicry leads to highly specialised pollination and provides a good system for investigating the process of pollinator-driven speciation. Here, we summarise the knowledge of key processes of speciation in this group of orchids and conduct a meta-analysis on traits that contribute to species differentiation, and thus potentially to speciation. Our study suggests that pollinator shift through changes in floral scent is predominant among closely related species in sexually deceptive orchids. Such shifts can provide a mechanism for pollinator-driven speciation in plants, if the resulting floral isolation is strong. Furthermore, changes in floral scent in these orchids are likely controlled by few genes. Together these factors suggest speciation in sexually deceptive orchids may happen rapidly and even in sympatry, which may explain the remarkable species diversity observed in this plant group.
\end{abstract}

\section{Introduction}

The species diversity in the orchid family is extraordinary. There are more than 20000 species in the orchid family [1], representing about 10 percent of angiosperm species. The remarkable floral forms found among orchids have always fascinated botanists and evolutionary biologists. Orchid species richness and the spectacular diversity of plantpollinator interactions provide an exceptional opportunity for the study of pollinator-driven speciation. There is more and more evidence suggesting that pollinators play a major role in the generation of novel floral forms and the maintenance of species diversity in orchids [2,3]. Sexually deceptive orchids are particularly suitable for investigating pollinatordriven speciation, because of their specialised pollination system. Here, using sexually deceptive orchids as examples, we systematically review what is known about the process of pollinator-driven speciation, examine the key factors that are essential for speciation processes, and discuss the possible modes of speciation in this orchid pollination system. In particular, we focus on the scenario of ecological speciation with gene flow.
Many theoretical analyses have suggested that three factors are of major importance in the process of ecological speciation: (i) disruptive selection, (ii) the strength of reproductive isolation, and (iii) the genetic basis of traits underlying reproductive isolation and traits under natural selection $[4,5]$. For reasons detailed below and discussed elsewhere [2], nonadaptive speciation is unlikely to play a major role in the diversification of the well-investigated genera of sexually deceptive orchids, Ophrys and Chiloglottis. In this review, we therefore focus on these three factors in sexually deceptive orchids.

\section{Sexually Deceptive Orchids}

Pollination by deception is very common in orchids (about one third of orchids are deceptive) $[1,6]$, with sexual deception as an especially intriguing variety. Sexually deceptive orchids mimic the mating signals of female insects and employ male insects as pollinators, inducing them to engage in mating behaviour and pseudocopulation [7-9]. The chemical mimicry of sex pheromones of pollinator 
females has been suggested to play a major role in this process [8-13]. Because insect mating signals are usually very specific [14], pollinator attraction by sexual deception is also very specific, each orchid only attracting one or very few insect species [15-17]. This specific pollinator attraction can therefore act as a reproductive barrier and prevent gene flow among species [7, 17-22].

Pollination by sexual deception is mostly found in orchids from Australia, Europe, South Africa, and South America [23-27]. However, sexual deception has recently been reported for the daisy Gorteria diffusa, the first confirmed case outside of Orchideceae [28], indicating that this pollination mechanism may be more common than previously thought [29]. Among the different sexually deceptive orchids, the two best-studied genera are Chiloglottis (Diurideae) in Australia, and Ophrys (Orchideae) in Europe. In Chiloglottis, there are more than 30 species that are pollinated mostly by thynnine wasps of the genus Neozeleboria [15, 30-32]. In Ophrys, most of the more than 200 species are pollinated by species representing different genera of solitary bees, while a few species are pollinated by species of solitary wasps, flies, and beetles [17, 33, 34].

Although sexually deceptive orchids have been suggested to be a spectacular example of ecological speciation (with or without gene flow), few studies have systematically investigated key factors like selection and reproductive isolation. Instead, most studies have focused on mechanistic aspects of floral mimicry $[8,9,35]$ or phylogenetic patterns $[36,37]$. Here, we critically review the process of ecological speciation with gene flow for two orchid systems with sexual mimicry. Additionally, we conduct a meta-analysis focusing on traits that differ among closely related orchid species. For our meta-study, we extracted and combined data from previous studies on orchid phylogeny $[22,37]$, pollinator information [34], and phenotypic traits [33, 38]. We considered only species for which all three types of information is available. Pairwise species comparisons of phenotypic traits and geographic distribution $[33,38]$ within monophyletic groups, both within Ophrys and Chiloglottis [22, 37], were performed, because reproductive isolation and selection is required to prevent gene flow within these groups. Since the potential for speciation through hybridisation has been reviewed previously [35], we will not discuss this aspect here.

\section{Pollinator-Mediated Selection in Sexually Deceptive Orchids}

In angiosperms, floral and species diversity is thought to be driven by pollinator-mediated selection because of pollen limitation [39]. Pollen limitation has been shown in most flowering plants [3, 39-42]. In orchids, many pollination studies suggest that pollen limitation is widespread in the whole family [24, 43-45]. This is especially true for deceptive orchids [46], because pollinator learning may reduce pollinator visitation $[47,48]$. Therefore, pollinators can impose strong selection on floral traits by mediating reproductive success and outcrossing [49]. Since morphology, sensory preferences, and behaviour differ among pollinator insects, selection imposed by them may lead to different adaptations $[21,49-51]$. Floral traits involved in plant-pollinator interactions, such as phenology, scent, colour, and morphology, are likely under pollinator-mediated selection, since they are directly associated with pollination success (Figure 1) [2]. However, only one study has hitherto quantified phenotypic selection in a sexually deceptive orchid [52]. Another interesting yet noninvestigated aspect is density-dependent selection, a potential driver of pollinator shifts in this group of plants. Below, we summarise the traits likely under selection and highlight important targets for future investigations.

Pollinator-mediated selection on floral phenology can be seen as the need for synchronisation between flowering time and emergence time of pollinators [23, 48]. Because of their pollinators' ability to learn to avoid deceptive flowers [53], most sexually deceptive orchids are probably predominantly pollinated by naïve male insects [54]. This may impose strong selection on the flowering time of orchids. In other words, the flowering time of different orchid species can be regarded as an adaptation to the optimal time of pollinator activity. From our meta-study, about $8-10 \%$ of closely related species pairs of sexually deceptive orchids did not overlap in floral phenology (counted from Table S1 available online at doi:10.1155/2012/285081), suggesting disruptive selection on floral phenology among closely related orchid species. However, most species pairs do overlap in flowering time, the average degree of flowering time overlap being 59\% and 47\%, in Ophrys and Chiloglottis, respectively (Table 1).

In most sexually deceptive orchids, floral scent, which mimics the sex pheromones of female pollinators, has been suggested to be the main attractant of pollinators $[8,9$, 20]; it is therefore likely to be under pollinator-mediated selection. Indeed, in both Ophrys and Chiloglottis, floral scent composition is driven towards their pollinators' preferences in both quantity and quality $[8,9,11-13,15,20,55]$. Furthermore, direct scent manipulation experiments showed that changing floral scent can dramatically reduce or increase pollinator visitation ([22] and Xu et al. unpublished data). Selection on floral scent is likely to be divergent among closely related species, which attract different pollinators with different floral scent compositions. In Chiloglottis, 95\% of closely related species pairs produce a different type of floral scent and are pollinated by different pollinators (Table 1). The same is true for Ophrys, where 98\% of closely related species pairs attract different pollinators (Table 1), most likely by the use of different floral odour bouquets $[12,17-$ 20, 55-59].

Despite the importance of floral scent, floral colour and/or floral display may also play a role for effective pollinator visitation. In many species of Ophrys and Chiloglottis, the floral labellum (a modified petal) resembles the visual pattern of pollinator females, indicating that pollinators may use this visual signal to locate the flowers at short distance $[38,48]$. Although it appears different to human eyes, the labellum of the Australian sexually deceptive orchid Cryptostylis is effectively identical in coloration to its pollinator females [60]. Many Ophrys labella may mimic the wings of a pollinator female with their shiny surface [16]. 


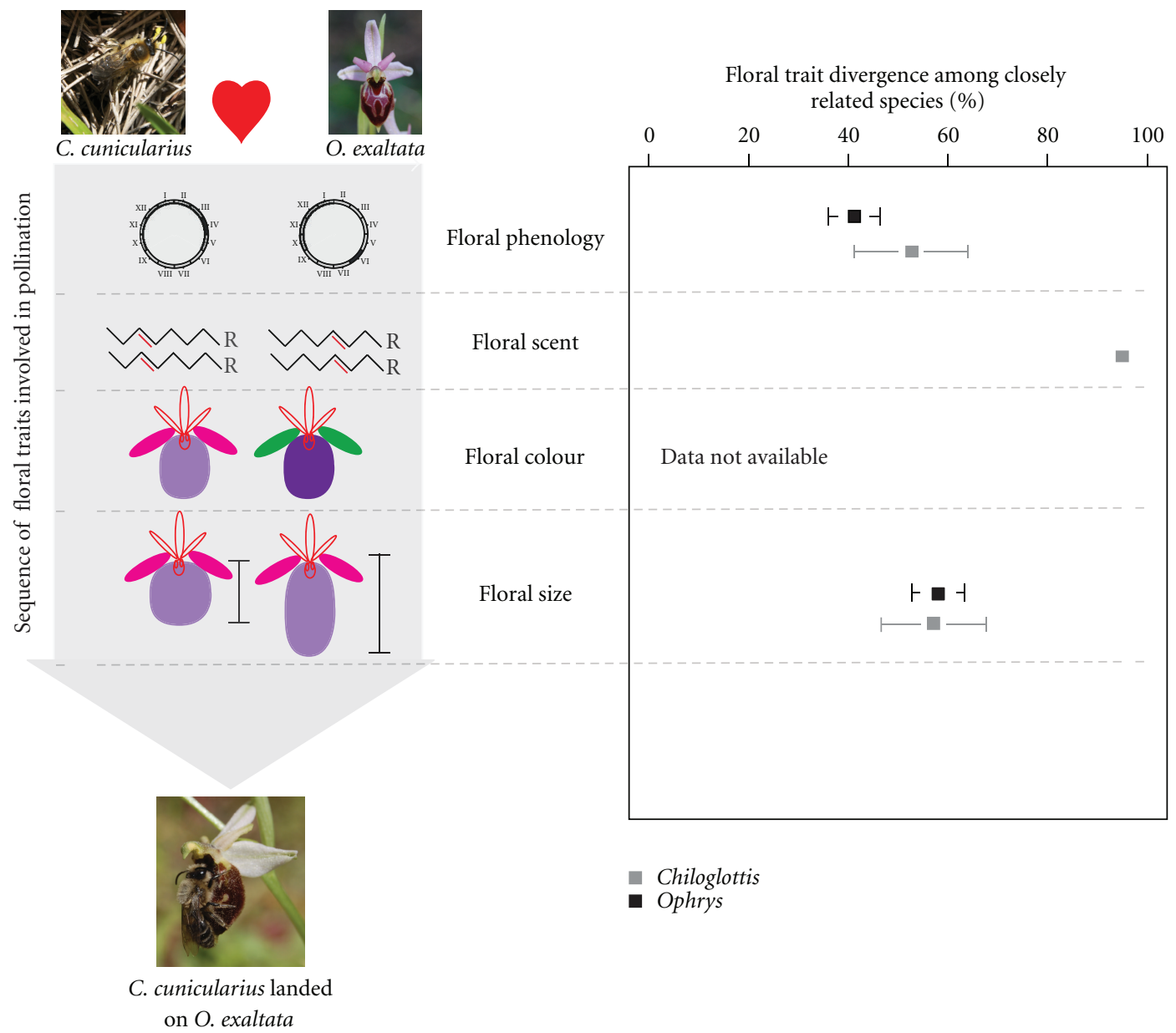

FIGURE 1: Summary of floral traits involved in plant-pollinator interaction. The left flow-chart shows the sequence of floral traits involved in pollination, whereas the right side shows the divergence of these traits between closely related species pairs in sexually deceptive orchids. Dark squares refer to Ophrys; grey squares to Chiloglottis. Floral trait data were extracted from Table 1 and Table S1; error bars represent standard error.

TABLE 1: Summary of comparisons of floral traits, distribution, and pollinators among closely related species pairs in Ophrys and Chiloglottis. Data on floral labellum, flowering time, and altitude range distribution are presented as mean ( \pm standard deviation). Data on distribution, floral scent, and pollinators are presented as mean value.

\begin{tabular}{|c|c|c|c|c|c|c|c|}
\hline Genus & $\begin{array}{c}\text { Number of } \\
\text { species-pairs } \\
\text { compared }\end{array}$ & $\begin{array}{c}\text { Overlap in labellum } \\
\text { length }(\%) \\
{[33,38]}\end{array}$ & $\begin{array}{c}\text { Overlap in } \\
\text { flowering time }(\%) \\
{[33,38]}\end{array}$ & $\begin{array}{c}\text { Overlap in habitat: } \\
\text { Altitude }(\%) \\
{[33,38]}\end{array}$ & $\begin{array}{c}\text { Overlap in } \\
\text { habitat: Area }(\%) \\
{[33,38]}\end{array}$ & $\begin{array}{c}\text { Overlap in floral } \\
\text { scent }(\%) \\
{[22]}\end{array}$ & $\begin{array}{c}\text { Overlap in } \\
\text { pollinators (\%) } \\
{[34]}\end{array}$ \\
\hline Ophrys & 213 & $42.6( \pm 31.7)$ & $59.0( \pm 31.2)$ & $83.0( \pm 19.2)$ & 53 & N/A & 2 \\
\hline Chiloglottis & 38 & $42.9( \pm 32.1)$ & $47.4( \pm 34.6)$ & $35.1( \pm 32.5)$ & 74 & 5 & 6 \\
\hline
\end{tabular}

In long-horned bee-pollinated Ophrys species, the perianth colour and/or contrast with background can affect the shortrange detectability of Ophrys flowers to their pollinators [6163]. However, this does not appear to be the case in a Colletes cunicularius-pollinated Ophrys species [57], suggesting that the role of colour and/or display on pollinator attraction may vary among different pollinators and visual systems. In our metastudy, we did not include floral colour, because most descriptions of floral coloration are based on observations by human eyes and not on insect vision models.
After the pollinator has landed on a flower, floral morphology such as labellum shape, size, and texture may have a strong influence on pollinator behaviour. In orchids, precisely removing and delivering pollinia is highly dependent on the match of shape and/or size of the pollinators' body and the floral labellum [48]. The significant correlation between pollinator body length and labellum length found in different Ophrys species clearly suggests that pollinatormediated selection on floral labellum size is strong [48, $52,64]$. Selection imposed on floral labellum size may 
be disruptive, since the body size of pollinators may vary considerably from each other. Indeed, the average overlap in floral labellum length among closely related species is only $43 \%$ in both Ophrys and Chiloglottis (Table 1), which suggests divergent selection on floral labellum size among closely related species.

\section{Reproductive Isolation in Sexually Deceptive Orchids}

Reproductive isolation among closely related sexually deceptive orchid species is mainly due to floral isolation, a form of pollinator-mediated prepollination reproductive isolation, while the postpollination reproductive barriers tend to be effectively absent or weak [21, 49] (but see [13]). For example, the closely related sympatric species $O$. exaltata, $O$. garganica, and $O$. sphegodes attract different solitary bees, Colletes cunicularius, Andrena pilipes, and Andrena nigroaenea, respectively, and the lack of pollinator sharing leads to a highly effective barrier to gene flow in this case, although interspecies hand pollination experiments yielded normal seed set [18]. Interestingly, this apparent lack of postpollination reproductive barriers appears to be common in both Ophrys and Chiloglottis $[22,65,66]$.

Since reproductive isolation in sexually deceptive orchids is mainly (if not only) mediated by floral isolation, the strength and stability of floral isolation are critical for pollinator-driven speciation. Although the hypothesis that floral isolation may effectively prevent gene flow between closely related species and thus drive speciation was already suggested half a century ago $[16,67]$, it was only recently that the strength of floral isolation was quantitatively measured in situ between species of the O. sphegodes group [18]. The results indicated that floral isolation among closely related species is very strong, with an isolation index higher than 0.98 [18]; a similar pattern was also found in another, unrelated Ophrys group (Gervasi and Schiestl, unpublished data), indicating that strong floral isolation is probably common in Ophrys. In Chiloglottis, pollinator behavioural tests on several sympatric species in different populations showed no cross-attraction, likewise suggesting strong floral isolation in this system $[30,66]$. Furthermore, population genetic studies in both Ophrys and Chiloglottis suggest that floral isolation can effectively prevent gene flow between species in sympatry $[18,19,66,68-70]$.

Other studies based on genetic and phylogenetic analysis showed low genetic divergence among species in Ophrys and concluded that floral isolation might not be strong enough to prevent gene flow between species in sympatry $[37,71$, 72]. However, low genetic divergence among species can be explained by different scenarios, such as recent radiations resulting in the retention of ancestral polymorphism [70]. Therefore, low genetic divergence among species cannot be taken as strong evidence of weak floral isolation per se. For a better understanding of the strength and variability of floral isolation, in situ measurements of floral isolation or systematic pollinator preference tests in more groups and populations over several years, or rigorous tests for hybridisation using molecular markers, are needed [73]. Furthermore, studies comparing neutral markers versus markers under disruptive selection will be important to shed light on the detailed mechanisms of speciation.

Floral isolation among closely related species of sexually deceptive orchids is either due to different pollinator attraction (ethological isolation) or different floral phenology (temporal isolation). Although mechanical isolation among species of the Ophrys sections Pseudophrys and Ophrys is evident $[67,74,75]$, these groups are not closely related to each other and thus mechanical (morphological) isolation is unlikely to play an important role in their radiation (see discussion below). The key trait involved in ethological isolation is floral scent $[21,22,56]$.

Ophrys flowers produce a complex mixture of more than 100 chemical compounds. Among these compounds, saturated and unsaturated hydrocarbons, that is, alkanes and alkenes, are responsible for pollinator attraction in many species $[9,11,12,18,76]$. Their specificity is due to quantitative variation in various alkenes with different double-bond positions and carbon chain lengths [9, 18, 19, 76, 77]. However, there are also some Ophrys species that employ only a few unusual chemical compounds for specific pollinator attraction. For example, in O. speculum, pollinator attraction can be achieved by the mixture of only eight compounds, in which two enantiomers of 9-hydroxydecanoic acid act as key substances [78]. Such signalling with few, specific chemical compounds is more common in Chiloglottis. Here, specific pollinator attraction is achieved by a single unusual compound (called "chiloglottone") [8] or simple blends of chemically related chiloglottones $[8,22]$.

Besides floral scent, floral phenology and floral morphology may also play a role in floral isolation among closely related species. Some species pairs show no overlap in flowering time in sympatry, (e.g., O. iricolor and $O$. mesaritica on Crete $[69,79])$. Among such species pairs, floral phenology may act as a strong reproductive barrier. However, as most closely related species pairs ( $90 \%)$ of sexually deceptive orchids do overlap in their flowering times to a certain degree, floral phenology alone is usually not the primary reproductive barrier. Differences in floral labellum length between closely related species pairs may contribute to mechanical reproductive isolation as well. However, due to pollinator movements during mating attempts, variation in labellum morphology alone might not provide a strong barrier to gene flow. To better understand the evolutionary patterns of the respective reproductive barriers in sexually deceptive orchids, direct quantitative measurements of the contributions of different floral traits to reproductive isolation are needed.

\section{The Genetic Basis of Reproductive "Barrier Traits" that Are under Pollinator- Mediated Divergent Selection}

Understanding the genetic basis and complexity of divergent traits involved in reproductive isolation is important for understanding the process of pollinator-driven speciation, 
since they basically constitute "automatic magic traits" [80]. Flowering time and flower morphology are likely to be complex genetic traits controlled by multilayered developmental genetic pathways that are still poorly understood in orchids [81]. Floral odour and coloration are likely formed by biosynthetic pathways that appear to be relatively conserved $[21,81]$. In both Ophrys and Chiloglottis, the pollinatorattractive scent compounds are probably derived from fatty acid intermediates $[35,77,82]$. Scent is a key trait for specific pollinator attraction and thus may be both important for reproductive isolation and subject to divergent selection, as changes in scent may directly cause pollinator shifts.

The number of genes underlying species differences in flower colour and odour has been suggested to be low [21]. For example, pollinator specificity among some species of Ophrys is linked to the double-bond position in a series of straight-chain alkenes, the bioactive pseudopheromone produced by the plants $[18,19]$. The alkenes are likely synthesised from few saturated (e.g., $\mathrm{C}_{16}$ and $\mathrm{C}_{18}$ ) fatty acids, and the introduction of the double-bond in these precursor compounds by stearoyl-ACP desaturase (SAD) enzymes is thought to lead to an orchestrated change in doublebond position in the downstream biosynthetic products [77]. Indeed, the alkene double-bond position in different Ophrys species is associated with changes in expression and possibly also activity of desaturases. For example, higher expression levels of SAD2 lead to the presence of higher amounts of 9- and 12-alkenes in Ophrys sphegodes [77]. Likewise, in sexually deceptive orchids with simple bioactive volatile chemistry such as Chiloglottis, a simple genetic basis underlying changes in scents and pollinator attraction has been hypothesised [22]. This seems especially plausible since most chiloglottones differ only in minor chemical modifications of a common structure. Thus, it seems that changes at few regulatory or structural genes are likely sufficient for changing traits that lead to changes in pollinator attraction in sexually deceptive orchids, favouring the view that changes in only a few genes of large effect rather than many genes of small effect can make speciation more likely. It should be noted, however, that although studies of molecular gene function may support this view, experiments to quantitatively assess the size of a gene's phenotypic effect in the field are necessary to test this hypothesis. For example, trait manipulation in the field or direct manipulation of gene expression via virus-induced gene silencing (VIGS) $[81,83]$ would allow testing the effect of a candidate gene on plantpollinator interactions. Moreover, another caveat is that the apparent simplicity of a candidate trait based on existing evidence may be misleading due to the inherent detection bias of current approaches.

\section{The Process of Pollinator-Driven Speciation in Orchids}

A key element of pollinator-driven speciation is that changes in pollinators are linked to reproductive isolation. While such pollinator shifts can in principle be brought about by a wide variety of floral trait changes, the importance of odour signals for pollinator attraction in sexually deceptive orchids makes floral scent the prime candidate for mediating pollinator shifts. Disruptive selection on odour phenotypes would lead to species divergence following an ecological speciation process $[84,85]$. Since such odour traits may have a simple genetic basis $[21,22,77,81]$, this may also be a process of speciation with gene flow (or, "genic speciation") [86-88]. Here, divergence occurs in spite of gene flow, while selection on a few loci in the genome is responsible for differences in pollinator attraction among species. The creation of population subdivision by pollinator-mediated selection may then lead to a buildup of larger islands of divergence in the genome brought about by an effective reduction of recombination, a process termed divergence hitchhiking [89-91]. At the same time, it should be noted that many orchid species have not reached the point where significant postmating barriers have accumulated [18, 65]. Overall, speciation by pollinator shift in sexually deceptive orchids may frequently be consistent with ecological speciation processes with gene flow.

The possibility for pollinator shifts is limited by the local availability of potential pollinators, which is subject to geographical variation, often referred to as the geographical pollinator mosaic [3]. The pollinator mosaic provides a route to allopatric and parapatric divergence scenarios [3], which are as applicable to sexually deceptive orchids as they are to other plants. However, unlike in many other pollination systems, sympatric speciation appears plausible in sexually deceptive systems $[17,19,20,22,35,69]$, the credibility of which is strengthened by the combination of strong floral isolation, divergent selection, and possibly a simple genic basis for species differences in this system. Molecular data suggesting gene flow among sympatric species (e.g., [72]) have been taken as evidence against pollinator-driven speciation in sympatry [3]. However, (i) it is often difficult to distinguish between current gene flow and ancestral polymorphism [92, 93], and (ii) gene flow itself is consistent with genic and ecological speciation processes [86-88]. This also highlights the difficulty researchers face to conclusively demonstrate sympatric speciation, requiring the combined study of population genetics (or genomics), phylogeny, biogeography, and data on the strength and genetic basis of reproductive barriers between sibling species.

Ecological speciation processes with gene flow are not only consistent with sympatric modes of speciation, but also the local nature of species divergence implies that speciation processes may often result in progenitor-derivative species patterns rather than strict sister species relationships. Progenitor-derivative speciation (or "quantum speciation") denotes the evolution of a new lineage (the derivative) from a source population (the progenitor) without affecting the progenitor [94-99]. Since pollinator shifts are not expected to change pollinator specificity in the source population, progenitor-derivative patterns are a likely outcome of pollinator-driven speciation in sexually deceptive orchids, with some genetic data supporting such scenarios $[69,70]$. Despite the potential for sympatric and progenitor-derivative speciation, it remains to be seen whether these modes of speciation are common in sexually deceptive orchids, 
our current knowledge being limited by the resolution of phylogenetic reconstructions among closely related sexually deceptive orchids (e.g., [37]).

\section{Conclusions and Future Directions}

Sexually deceptive orchids provide an exceptional study system for pollinator-driven speciation in plants. Our critical review and meta-analysis of traits in closely related sexually deceptive orchid species pairs suggest that pollinator shifts through changes in floral traits, especially floral scent, may be the main mechanism for speciation in this plant group. Such speciation through pollinator shift may happen rapidly and even in sympatry, if floral isolation is strong and floral trait changes are controlled by few genes. Therefore, to understand the detailed process of speciation in sexually deceptive orchids, it is necessary to identify the genetic basis of key floral traits and quantify their contributions to plantpollinator interaction. Furthermore, to better understand the speciation patterns in this plant group, it would be important to evaluate their likelihood in a theoretical model under biologically plausible conditions based on empirical data $[100,101]$. Such an integrative theoretical framework is still missing but is urgently needed to truly understand pollinator-driven speciation.

\section{Acknowledgments}

The authors are grateful for the financial support by ETH Zürich (TH0206-2 to FPS) and Swiss National Science Foundation (SNF 31003A_130796 to PMS). The authors thank R. Faria and two anonymous reviewers for their constructive comments on the paper.

\section{References}

[1] L. R. Dressler, The Orchids: Natural History and Classification, Harvard University Press, Cambridge, Mass, USA, 1981.

[2] F. P. Schiestl, "Animal pollination and speciation in plants: general mechanisms and examples from the orchids," in Evolution of Plant Pollinator Interactions, S. Patiny, Ed., Cambridge University Press, New York, NY, USA.

[3] S. D. Johnson, "Pollinator driven speciation in plants," in Ecology and Evolution of Flowers, L. D. Harder and S. C. H. Barrett, Eds., pp. 295-310, Oxford University Press, Oxford, UK, 2006.

[4] N. M. Waser and D. R. Campbell, "Ecological speciation in flowering plants," in Adaptive Speciation, U. Dieckmann, M. Doebeli, M. J. Metz, and D. Tautz, Eds., pp. 264-277, Cambridge University Press, Cambridge, UK, 2004.

[5] J. A. Coyne and H. A. Orr, Speciation, Sinauer Associates, Sunderland, Mass, USA, 2004.

[6] S. Cozzolino and A. Widmer, "Orchid diversity: an evolutionary consequence of deception?" Trends in Ecology and Evolution, vol. 20, no. 9, pp. 487-494, 2005.

[7] R. Peakall, "Responses of male Zaspilothynnus trilobatus Turner wasps to females and the sexually deceptive orchid it pollinates," Functional Ecology, vol. 4, no. 2, pp. 159-167, 1990.
[8] F. P. Schiestl, R. Peakall, J. G. Mant et al., "The chemistry of sexual deception in an orchid-wasp pollination system," Science, vol. 302, no. 5644, pp. 437-438, 2003.

[9] F. P. Schiestl, M. Ayasse, H. F. Paulus et al., "Orchid pollination by sexual swindle," Nature, vol. 399, no. 6735, pp. 421-422, 1999.

[10] M. Ayasse, F. P. Schiestl, H. F. Paulus, D. Erdmann, and W. Francke, "Chemical communication in the reproductive biology of Ophrys sphegodes," Mitteilungen Der Deutschen Gesellschaft Für Allgemeine Und Agewandte Entomologie, vol. 11, no. 1-6, pp. 473-476, 1997.

[11] J. Stökl, R. Twele, D. H. Erdmann, W. Francke, and M. Ayasse, "Comparison of the flower scent of the sexually deceptive orchid Ophrys iricolor and the female sex pheromone of its pollinator Andrena morio," Chemoecology, vol. 17, no. 4, pp. 231-233, 2007.

[12] J. Gögler, J. Stökl, A. Sramkova et al., "The role of pollinator attracting scent in the sexually deceptive orchids Ophrys chestermanii, O. normanii and O. tenthredinifera," Mitteilungen Der Deutschen Gesellschaft Für Allgemeine Und Angewandte Entomologie, vol. 16, pp. 175-178, 2008.

[13] J. Gögler, J. Stökl, A. Sramkova et al., "Ménage à troistwo endemic species of deceptive orchids and one pollinator species," Evolution, vol. 63, no. 9, pp. 2222-2234, 2009.

[14] M. Ayasse, R. J. Paxton, and J. Tengö, "Mating behavior and chemical communication in the order Hymenoptera," Annual Review of Entomology, vol. 46, pp. 31-78, 2001.

[15] C. C. Bower and G. R. Brown, "Pollinator specificity, cryptic species and geographical patterns in pollinator responses to sexually deceptive orchids in the genus Chiloglottis: the Chiloglottis gunnii complex," Australian Journal of Botany, vol. 57, no. 1, pp. 37-55, 2009.

[16] B. Kullenberg, "Studies in Ophrys pollination," Zoologiska Bidrag Från Uppsala, vol. 34, pp. 1-340, 1961.

[17] H. F. Paulus and C. Gack, "Pollinators as prepollinating isolation factors: evolution and speciation in Ophrys (Orchidaceae)," Israel Journal of Botany, vol. 39, no. 1-2, pp. 43-79, 1990.

[18] S. Xu, P. M. Schlüter, G. Scopece et al., "Floral isolation is the main reproductive barrier among closely related sexually deceptive orchids," Evolution, vol. 65, no. 9, pp. 2606-2620, 2011.

[19] J. Mant, R. Peakall, and F. P. Schiestl, "Does selection on floral odor promote differentiation among populations and species of the sexually deceptive orchid genus Ophrys?" Evolution, vol. 59, no. 7, pp. 1449-1463, 2005.

[20] F. P. Schiestl and M. Ayasse, "Do changes in floral odor cause speciation in sexually deceptive orchids?" Plant Systematics and Evolution, vol. 234, no. 1-4, pp. 111-119, 2002.

[21] F. P. Schiestl and P. M. Schlüter, "Floral isolation, specialized pollination, and pollinator behavior in orchids," Annual Review of Entomology, vol. 54, pp. 425-446, 2009.

[22] R. Peakall, D. Ebert, J. Poldy et al., "Pollinator specificity, floral odour chemistry and the phylogeny of Australian sexually deceptive Chiloglottis orchids: implications for pollinatordriven speciation," New Phytologist, vol. 188, no. 2, pp. 437450, 2010.

[23] A. Dafni, "Mimicry and deception in pollination," Annual Review of Ecology and Systematics, vol. 15, pp. 259-278, 1984.

[24] L. A. Nilsson, "Orchid pollination biology," Trends in Ecology and Evolution, vol. 7, no. 8, pp. 255-259, 1992.

[25] K. E. Steiner, V. B. Whitehead, and S. D. Johnson, "Floral and pollinator divergence in two sexually deceptive South African 
orchids," American Journal of Botany, vol. 81, no. 2, pp. 185194, 1994.

[26] R. B. Singer, "The pollination mechanism in Trigonidium obtusum Lindl (Orchidaceae: Maxillariinae): sexual mimicry and trap-flowers," Annals of Botany, vol. 89, no. 2, pp. 157$163,2002$.

[27] R. B. Singer, A. Flach, S. Koehler, A. J. Marsaioli, and M. D. C. E. Amaral, "Sexual mimicry in Mormolyca ringens (Lindl.) Schltr. (Orchidaceae: Maxillariinae)," Annals of Botany, vol. 93, no. 6, pp. 755-762, 2004.

[28] A. G. Ellis and S. D. Johnson, "Floral mimicry enhances pollen export: the evolution of pollination by sexual deceit outside of the Orchidaceae," The American Naturalist, vol. 176, no. 5, pp. E143-E151, 2010.

[29] F. P. Schiestl, "Pollination: sexual mimicry abounds," Current Biology, vol. 20, no. 23, pp. R1020-R1022, 2010.

[30] C. C. Bower, "Demonstration of pollinator-mediated reproductive isolation in sexually deceptive species of Chiloglottis (Orchidaceae: Caladeniinae)," Australian Journal of Botany, vol. 44, no. 1, pp. 15-33, 1996.

[31] C. C. Bower, "Specific pollinators reveal a cryptic taxon in the bird orchid, Chiloglottis valida sensu lato (Orchidaceae) in south-eastern Australia," Australian Journal of Botany, vol. 54, no. 1, pp. 53-64, 2006.

[32] J. Mant, R. Peakall, and P. H. Weston, "Specific pollinator attraction and the diversification of sexually deceptive Chiloglottis (Orchidaceae)," Plant Systematics and Evolution, vol. 253, no. 1-4, pp. 185-200, 2005.

[33] P. Delforge, Orchids of Europe, North Africa, and the Middle East, A\&C Black, London, UK, 3rd edition, 2006.

[34] A. C. Gaskett, "Orchid pollination by sexual deception: pollinator perspectives," Biological Reviews, vol. 86, no. 1, pp. 33-75, 2011.

[35] M. Ayasse, J. Stökl, and W. Francke, "Chemical ecology and pollinator-driven speciation in sexually deceptive orchids," Phytochemistry, vol. 72, no. 13, pp. 1667-1677, 2011.

[36] M. Soliva, A. Kocyan, and A. Widmer, "Molecular phylogenetics of the sexually deceptive orchid genus Ophrys (Orchidaceae) based on nuclear and chloroplast DNA sequences," Molecular Phylogenetics and Evolution, vol. 20, no. 1, pp. 7888, 2001.

[37] D. S. Devey, R. M. Bateman, M. F. Fay, and J. A. Hawkins, "Friends or relatives? Phylogenetics and species delimitation in the controversial European orchid genus Ophrys," Annals of Botany, vol. 101, no. 3, pp. 385-402, 2008.

[38] D. L. Jones, A Complete Guide to Native Orchids of Australia Including the Island Territories, Reed New Holland, Sydney, Australia, 2006.

[39] T. M. Knight, J. A. Steets, J. C. Vamosi et al., "Pollen limitation of plant reproduction: pattern and process," Annual Review of Ecology, Evolution, and Systematics, vol. 36, pp. 467-497, 2005.

[40] M. Burd, "Bateman's principle and plant reproduction: the role of pollen limitation in fruit and seed set," Botanical Review, vol. 60, no. 1, pp. 83-139, 1994.

[41] T. L. Ashman, T. M. Knight, J. A. Steets et al., "Pollen limitation of plant reproduction: ecological and evolutionary causes and consequences," Ecology, vol. 85, no. 9, pp. 24082421, 2004.

[42] L. D. Harder and M. A. Aizen, "Floral adaptation and diversification under pollen limitation," Philosophical Transactions of the Royal Society B, vol. 365, no. 1539, pp. 529-543, 2010.
[43] S. D. Johnson and W. J. Bond, "Evidence for widespread pollen limitation of fruiting success in Cape wildflowers," Oecologia, vol. 109, no. 4, pp. 530-534, 1997.

[44] J. D. Ackerman and A. M. Montalvo, "Short- and long-term limitations to fruit production in a tropical orchid," Ecology, vol. 71, no. 1, pp. 263-272, 1990.

[45] L. M. O'Connell and M. O. Johnston, "Male and female pollination success in a deceptive orchid, a selection study," Ecology, vol. 79, no. 4, pp. 1246-1260, 1998.

[46] G. Scopece, S. Cozzolino, S. D. Johnson, and F. P. Schiestl, "Pollination efficiency and the evolution of specialized deceptive pollination systems," The American Naturalist, vol. 175, no. 1, pp. 98-105, 2010.

[47] J. B. Ferdy, P. H. Gouyon, J. Moret, and B. Godelle, "Pollinator behavior and deceptive pollination: learning process and floral evolution," The American Naturalist, vol. 152, no. 5, pp. 696-705, 1998.

[48] H. F. Paulus, "Deceived males-Pollination biology of the Mediterranean orchid genus Ophrys (Orchidaceae)," Journal Europäischer Orchideen, vol. 38, no. 2, pp. 303-353, 2006.

[49] K. M. Kay and R. D. Sargent, "The role of animal pollination in plant speciation: integrating ecology, geography, and genetics," Annual Review of Ecology, Evolution, and Systematics, vol. 40, pp. 637-656, 2009.

[50] G. L. Stebbins, "Adaptive radiation of reproductive characteristics in angiosperms, I: pollination mechanisms," Annual Review of Ecology, Evolution, and Systematics, vol. 1, no. 1, pp. 307-326, 1970.

[51] R. D. Sargent and S. P. Otto, "The role of local species abundance in the evolution of pollinator attraction in flowering plants," The American Naturalist, vol. 167, no. 1, pp. 67-80, 2006.

[52] S. Benitez-Vieyra, A. M. Medina, and A. A. Cocucci, "Variable selection patterns on the labellum shape of Geoblasta pennicillata, a sexually deceptive orchid," Journal of Evolutionary Biology, vol. 22, no. 11, pp. 2354-2362, 2009.

[53] M. Ayasse, F. P. Schiestl, H. F. Paulus et al., "Evolution of reproductive strategies in the sexually deceptive Orchid Ophrys sphegodes: how does flower-specific variation of odor signals influence reproductive success?" Evolution, vol. 54, no. 6, pp. 1995-2006, 2000.

[54] H. F. Paulus, "Co-evolution and unilateral adaptation in flower-pollinator systems: pollinators as pacemakers in the evolution of flowers," Verhandlungen Der Deutschen Zoologischen Gesellschaft, vol. 81, pp. 25-46, 1988.

[55] J. Stökl, P. M. Schlüter, T. F. Stuessy et al., "Speciation in sexually deceptive orchids: pollinator-driven selection maintains discrete odour phenotypes in hybridizing species," Biological Journal of the Linnean Society, vol. 98, no. 2, pp. 439-451, 2009.

[56] F. P. Schiestl, "On the success of a swindle: pollination by deception in orchids," Die Naturwissenschaften, vol. 92, no. 6, pp. 255-264, 2005.

[57] N. J. Vereecken and F. P. Schiestl, "On the roles of colour and scent in a specialized floral mimicry system," Annals of Botany, vol. 104, no. 6, pp. 1077-1084, 2009.

[58] A. K. Borg-Karlson and J. Tengö, "Odor mimetism? Key substances in Ophrys lutea-Andrena pollination relationship (Orchidaceae: Andrenidae)," Journal of Chemical Ecology, vol. 12, no. 9, pp. 1927-1941, 1986.

[59] N. J. Vereecken, S. Cozzolino, and F. P. Schiestl, "Hybrid floral scent novelty drives pollinator shift in sexually deceptive orchids," BMC Evolutionary Biology, vol. 10, no. 1, article 103, 2010 . 
[60] A. C. Gaskett and M. E. Herberstein, "Colour mimicry and sexual deception by Tongue orchids (Cryptostylis)," Naturwissenschaften, vol. 97, no. 1, pp. 97-102, 2010.

[61] M. Streinzer, T. Ellis, H. F. Paulus, and J. Spaethe, "Visual discrimination between two sexually deceptive Ophrys species by a bee pollinator," Arthropod-Plant Interactions, vol. 4, no. 3, pp. 141-148, 2010.

[62] M. Streinzer, H. F. Paulus, and J. Spaethe, "Floral colour signal increases short-range detectability of a sexually deceptive orchid to its bee pollinator," Journal of Experimental Biology, vol. 212, no. 9, pp. 1365-1370, 2009.

[63] J. Spaethe, W. H. Moser, and H. F. Paulus, "Increase of pollinator attraction by means of a visual signal in the sexually deceptive orchid, Ophrys heldreichii (Orchidaceae)," Plant Systematics and Evolution, vol. 264, no. 1-2, pp. 31-40, 2007.

[64] N. J. Vereecken, "Deceptive behavior in plants. I. Pollination by sexual deception in orchids: a host-parasite perspective," in Plant-Environment Interactions, F. Baluska, Ed., pp. 203222, Springer, Heidelberg, Germany, 2009.

[65] G. Scopece, A. Musacchio, A. Widmer, and S. Cozzolino, "Patterns of reproductive isolation in Mediterranean deceptive orchids," Evolution, vol. 61, no. 11, pp. 2623-2642, 2007.

[66] J. Mant, C. C. Bower, P. H. Weston, and R. Peakall, "Phylogeography of pollinator-specific sexually deceptive Chiloglottis taxa (Orchidaceae): evidence for sympatric divergence?" Molecular Ecology, vol. 14, no. 10, pp. 3067-3076, 2005.

[67] B. Kullenberg, "Investigations on the pollination of Ophrys species," Oikos, vol. 2, no. 1, pp. 1-19, 1950.

[68] P. M. Schlüter, P. M. Ruas, G. Kohl, C. F. Ruas, T. F. Stuessy, and H. F. Paulus, "Reproductive isolation in the Aegean Ophrys omegaifera complex (Orchidaceae)," Plant Systematics and Evolution, vol. 267, no. 1-4, pp. 105-119, 2007.

[69] P. M. Schlüter, P. M. Ruas, G. Kohl, C. F. Ruas, T. F. Stuessy, and H. F. Paulus, "Genetic patterns and pollination in Ophrys iricolor and O. mesaritica (Orchidaceae): sympatric evolution by pollinator shift," Botanical Journal of the Linnean Society, vol. 159, no. 4, pp. 583-598, 2009.

[70] P. M. Schlüter, P. M. Ruas, G. Kohl, C. F. Ruas, T. F. Stuessy, and H. F. Paulus, "Evidence for progenitor-derivative speciation in sexually deceptive orchids," Annals of Botany, vol. 108, pp. 895-906, 2011.

[71] D. S. Devey, R. M. Bateman, M. F. Fay, and J. A. Hawkins, "Genetic structure and systematic relationships within the Ophrys fuciflora aggregate (Orchidaceae: Orchidinae): high diversity in Kent and a wind-induced discontinuity bisecting the Adriatic," Annals of Botany, vol. 104, no. 3, pp. 483-495, 2009.

[72] M. Soliva and A. Widmer, "Gene flow across species boundaries in sympatric, sexually deceptive Ophrys (Orchidaceae) species," Evolution, vol. 57, no. 10, pp. 2252-2261, 2003.

[73] R. Peakall, C. C. Bower, A. E. Logan, and H. I. Nicol, "Confirmation of the hybrid origin of Chiloglottis $x$ pescottiana (Orchidaceae: Diurideae). 1. Genetic and morphometric evidence," Australian Journal of Botany, vol. 45, no. 5, pp. 839-855, 1997.

[74] L. Ågren, B. Kullenberg, and T. Sensenbaugh, "Congruences in pilosity between three species of Ophrys (Orchidaceae) in their hymenopteran pollinators," Nova Acta Regiae Societatis Scientiarum Upsaliensis, Serie V, vol. 3, no. 3, pp. 15-25, 1984.

[75] P. Cortis, N. J. Vereecken, F. P. Schiestl, M. R. Barone Lumaga, A. Scrugli, and S. Cozzolino, "Pollinator convergence and the nature of species' boundaries in sympatric Sardinian Ophrys
(Orchidaceae)," Annals of Botany, vol. 104, no. 3, pp. 497$506,2009$.

[76] J. Mant, C. Brändli, N. J. Vereecken, C. M. Schulz, W. Francke, and F. P. Schiestl, "Cuticular hydrocarbons as sex pheromone of the bee Colletes cunicularius and the key to its mimicry by the sexually deceptive orchid, Ophrys exaltata," Journal of Chemical Ecology, vol. 31, no. 8, pp. 1765-1787, 2005.

[77] P. M. Schlüter, S. Xu, V. Gagliardini et al., "Stearoyl-acyl carrier protein desaturases are associated with floral isolation in sexually deceptive orchids," Proceedings of the National Academy of Sciences of the United States of America, vol. 108, no. 14 , pp. 5696-5701, 2011.

[78] M. Ayasse, F. P. Schiestl, H. F. Paulus, F. Ibarra, and W. Francke, "Pollinator attraction in a sexually deceptive orchid by means of unconventional chemicals," Proceedings of the Royal Society B, vol. 270, no. 1514, pp. 517-522, 2003.

[79] H. Kretzschmar, G. Kretzschmar, and W. Eccarius, Orchideen auf Kreta, Kasos und Karpathos. Ein Feldführer durch die Orchideenflora der zentralen Insel der Südägäis, Selbstverlag H. Kretzschmar, Bad Hersfeld, Germany, 2002.

[80] M. R. Servedio, G. S. Van Doorn, M. Kopp, A. M. Frame, and P. Nosil, "Magic traits in speciation: "magic" but not rare?" Trends in Ecology \& Evolution, vol. 26, no. 8, pp. 389-397, 2011.

[81] P. M. Schlüter and F. P. Schiestl, "Molecular mechanisms of floral mimicry in orchids," Trends in Plant Science, vol. 13, no. 5, pp. 228-235, 2008.

[82] S. Franke, F. Ibarra, C. M. Schulz et al., "The discovery of 2,5-dialkylcyclohexan-1,3-diones as a new class of natural products," Proceedings of the National Academy of Sciences of the United States of America, vol. 106, no. 22, pp. 8877-8882, 2009.

[83] T. M. Burch-Smith, J. C. Anderson, G. B. Martin, and S. P. Dinesh-Kumar, "Applications and advantages of virusinduced gene silencing for gene function studies in plants," Plant Journal, vol. 39, no. 5, pp. 734-746, 2004.

[84] T. J. Givnish, "Ecology of plant speciation," Taxon, vol. 59, no. 5, pp. 1329-1366, 2010.

[85] D. Schluter and G. L. Conte, "Genetics and ecological speciation," Proceedings of the National Academy of Sciences of the United States of America, vol. 106, pp. 9955-9962, 2009.

[86] C. I. Wu, "The genic view of the process of speciation," Journal of Evolutionary Biology, vol. 14, no. 6, pp. 851-865, 2001.

[87] C. Lexer and A. Widmer, "The genic view of plant speciation: recent progress and emerging questions," Philosophical Transactions of the Royal Society B, vol. 363, no. 1506, pp. 3023-3036, 2008.

[88] C. I. Wu and C. T. Ting, "Genes and speciation," Nature Reviews Genetics, vol. 5, no. 2, pp. 114-122, 2004.

[89] S. Via, "Natural selection in action during speciation," Proceedings of the National Academy of Sciences of the United States of America, vol. 106, pp. 9939-9946, 2009.

[90] S. Via and J. West, "The genetic mosaic suggests a new role for hitchhiking in ecological speciation," Molecular Ecology, vol. 17, no. 19, pp. 4334-4345, 2008.

[91] J. L. Feder and P. Nosil, "The efficacy of divergence hitchhiking in generating genomic islands during ecological speciation," Evolution, vol. 64, no. 6, pp. 1729-1747, 2010.

[92] D. J. Funk and K. E. Omland, "Species-level paraphyly and polyphyly: frequency, causes, and consequences, with insights from animal mitochondrial DNA," Annual Review of Ecology, Evolution, and Systematics, vol. 34, pp. 397-423, 2003. 
[93] C. Pinho and J. Hey, "Divergence with gene flow: models and data," Annual Review of Ecology, Evolution, and Systematics, vol. 41, pp. 215-230, 2010.

[94] D. A. Levin, "Local speciation in plants: the rule not the exception," Systematic Botany, vol. 18, no. 2, pp. 197-208, 1993.

[95] D. A. Levin, “The ecological transition in speciation," New Phytologist, vol. 161, no. 1, pp. 91-96, 2004.

[96] L. H. Rieseberg and L. Brouillet, "Are many plant species paraphyletic?" Taxon, vol. 43, no. 1, pp. 21-32, 1994.

[97] D. J. Crawford, "Progenitor-derivative species pairs and plant speciation," Taxon, vol. 59, no. 5, pp. 1413-1423, 2010.

[98] L. D. Gottlieb, "Rethinking classic examples of recent speciation in plants," New Phytologist, vol. 161, no. 1, pp. 71-82, 2004.

[99] V. Grant, Plant Speciation, Columbia University Press, New York, NY, USA, 2nd edition, 1981.

[100] D. I. Bolnick and B. M. Fitzpatrick, "Sympatric speciation: models and empirical evidence," Annual Review of Ecology, Evolution, and Systematics, vol. 38, pp. 459-487, 2007.

[101] D. I. Bolnick, "Sympatric speciation in threespine stickleback: why not?" International Journal of Ecology, vol. 2011, Article ID 942847, 15 pages, 2011. 

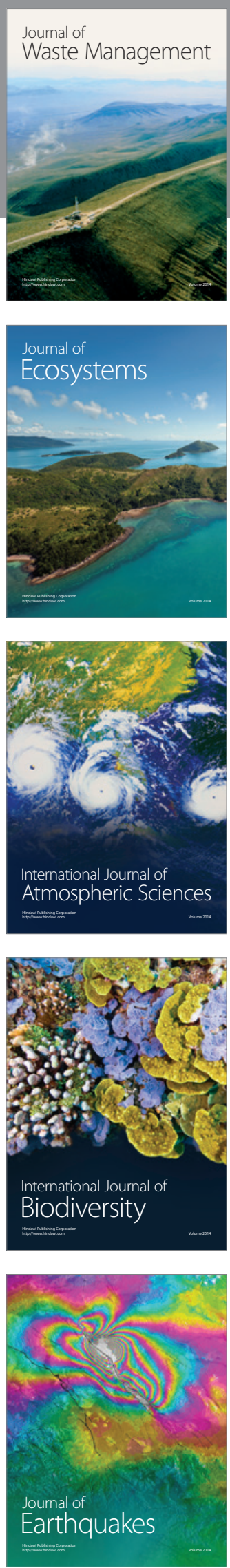
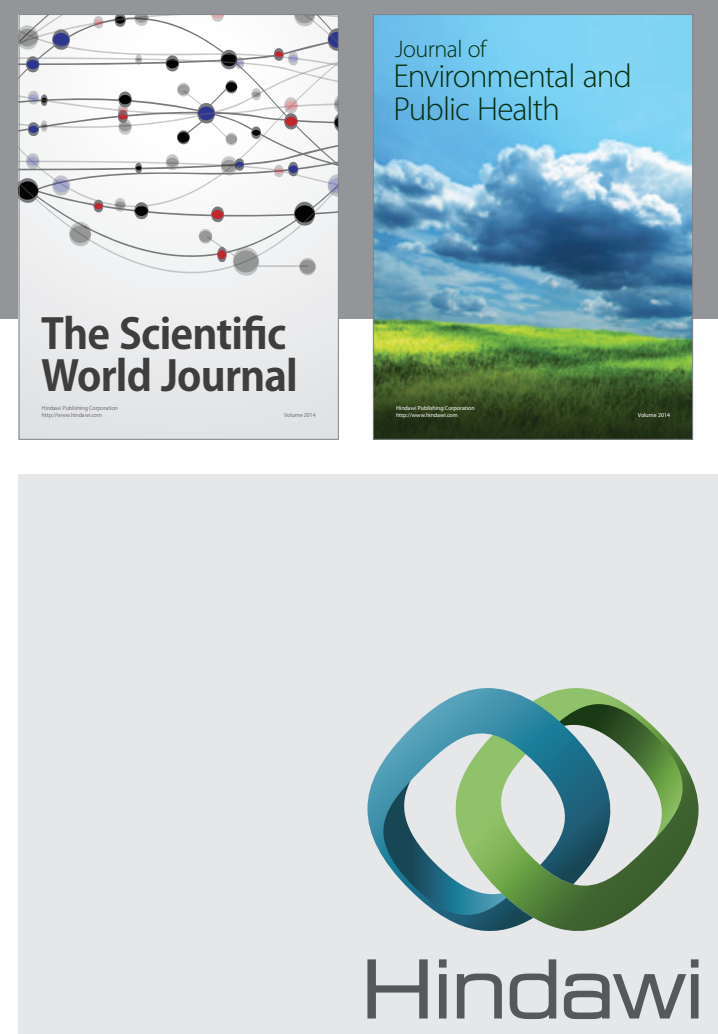

Submit your manuscripts at

http://www.hindawi.com
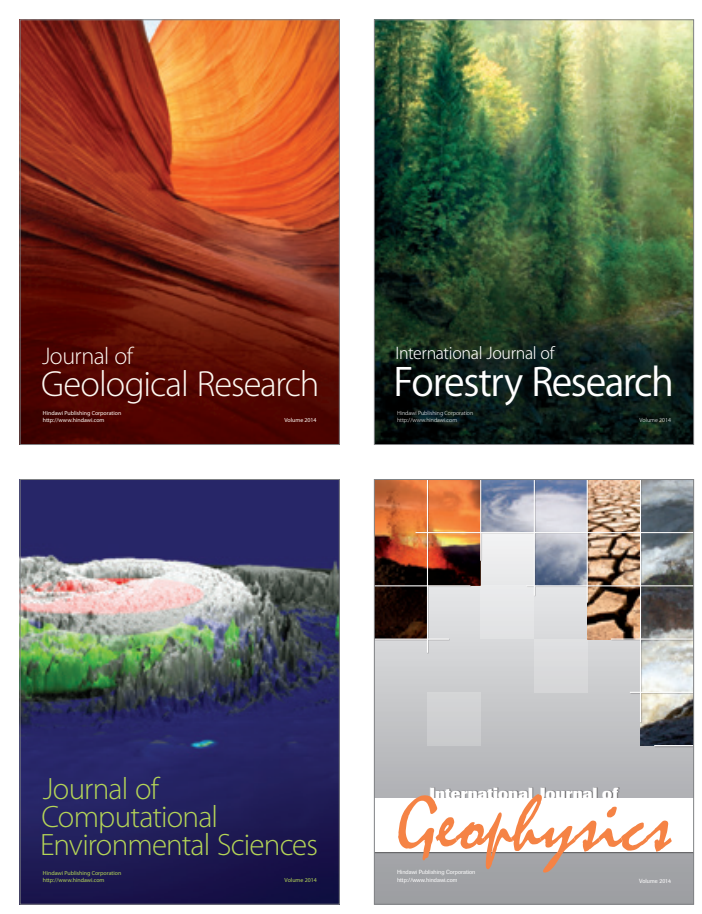
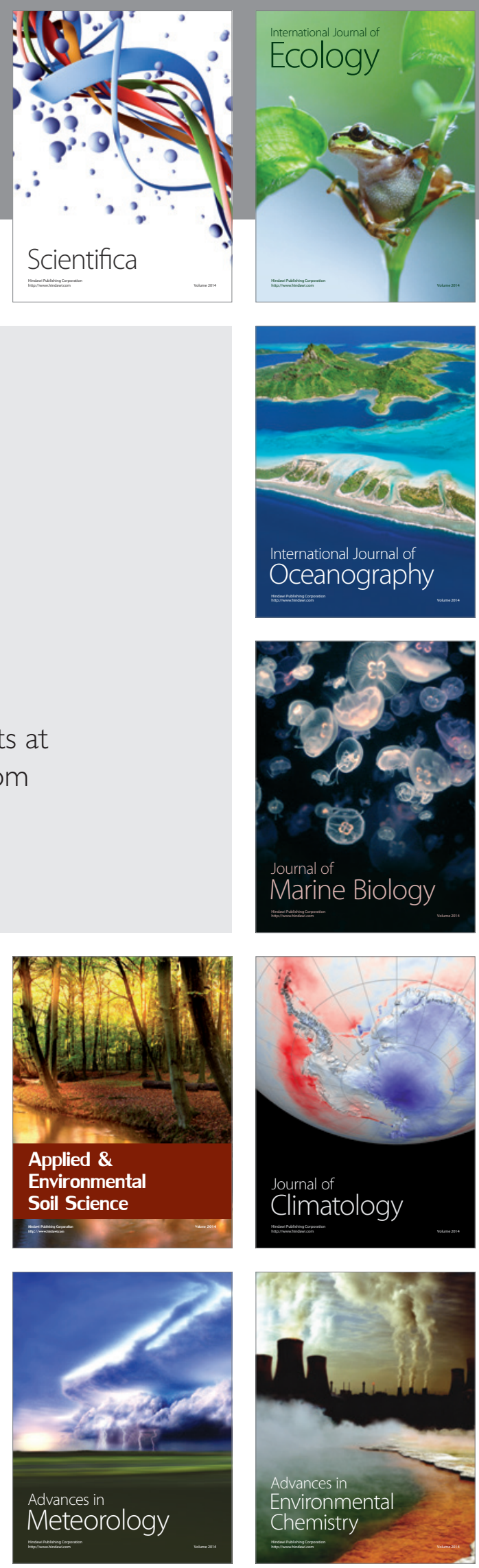